(C) 2017 IEEE. Personal use of this material is permitted. Permission from IEEE must be obtained for all other uses, in any current or future media, including reprinting/republishing this material for advertising or promotional purposes, creating new collective works, for resale or redistribution to servers or lists, or reuse of any copyrighted component of this work in other works. 


\title{
Detection of Turning Freeze in Parkinson's Disease based on S-transform Decomposition of EEG signals
}

\author{
Quynh Tran Ly ${ }^{\mathrm{a}}$, Student Member, IEEE, A.M. Ardi Handojoseno ${ }^{\mathrm{a}, \mathrm{c}, \dagger}$, Moran Gilat ${ }^{\mathrm{b}}$, Rifai Chai ${ }^{\mathrm{a}}$, \\ Kaylena A. Ehgoetz Martens ${ }^{\mathrm{b}}$, Matthew Georgiades ${ }^{\mathrm{b}}$, Member, IEEE, Ganesh R. Naik $^{\mathrm{a}}$, Yvonne Tran ${ }^{\mathrm{a}}$,

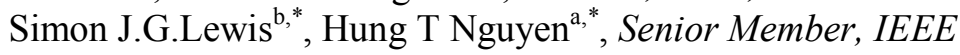

\begin{abstract}
Freezing of Gait (FOG) is a highly debilitating and poorly understood symptom of Parkinson's disease (PD), causing severe immobility and decreased quality of life. Turning Freezing (TF) is known as the most common sub-type of FOG, also causing the highest rate of falls in PD patients. During a TF, the feet of PD patients appear to become stuck whilst making a turn. This paper presents an electroencephalography (EEG) based classification method for detecting turning freezing episodes in six PD patients during Timed Up and Go Task experiments. Since EEG signals have a time-variant nature, time-frequency Stockwell Transform (STransform) techniques were used for feature extraction. The EEG sources were separated by means of independent component analysis using entropy bound minimization (ICAEBM). The distinctive frequency-based features of selected independent components of EEG were extracted and classified using Bayesian Neural Networks. The classification demonstrated a high sensitivity of $84.2 \%$, a specificity of $88.0 \%$ and an accuracy of $86.2 \%$ for detecting TF. These promising results pave the way for the development of a real-time device for detecting different sub-types of FOG during ambulation.
\end{abstract}

\section{INTRODUCTION}

Freezing of gait (FOG) are episodes where Parkinson's disease (PD) patients suddenly become unable to walk and often feel as though their feet have been 'glued' to the ground. Based on contexts of behavioral measures, different types of FOG have been observed including: start hesitation (Gait Initiation Failure), freezing whilst passing through narrow gaps, target hesitation, freezing when dual-tasking, freezing on an open runway and turning freezing (TF) [1]. TF is defined as the phenomenon in which the feet of PD patients appear to become stuck whilst usually making a tight turn. Importantly, $\mathrm{TF}$ is recognized to be the most frequent trigger of FOG, totaling $62.7 \%$ of all witnessed FOG episodes [1]. Turning is a complex motor task requiring motor and cognitive processing to support the correct selection, timing and scaling of movement. TF has clinical importance as it increases the risk for falls and falls related to injuries [1]. However, the pathophysiological basis for these effects is not well known [2].

\footnotetext{
${ }^{a}$ Faculty of Engineering and Information Technology, University of Technology, Sydney, Broadway, NSW 2007, Australia.

b Parkinson's Disease Research Clinic, Brain and Mind Center, University of Sydney, Level 4, Building F, 94 Mallet Street, Camper down, NSW, 2050, Australia.

c Faculty of Science and Engineering, Sanata Dharma University, Paingan, Sleman, Yogyakarta, 55281, Indonesia.

${ }^{*}$ Dual senior authorship.

${ }^{\dagger}$ Deceased April 8, 2017.
}

Our group has previously developed an algorithm that could detect TF by analyzing energy power and brain effective connectivity of EEG signals with an averaged sensitivity around at 68\% [3]. This EEG approach offers the potential to identify and detect freezing during turning due to its ability to track the dynamic physiological changes through the brain in real time. In addition, optimal EEG detection would allow for treatment options to be implemented in a timely manner to overcome and preferably prevent TF. As a result, it is critical that these detection methods will need to obtain high classification accuracies. With the goal of improving classification accuracy, the current paper aims to further explore the computational intelligence for EEG signals during TF by applying source separation technique (independent component analysis by entropy bound minimization, ICA-EBM) and S-Transform decomposition as feature extraction methods. The entropy bound estimation in ICA-EBM was chosen for its flexibility and its ability to approximate sources of a wide range of distributions that fit with EEG signals; which indeed improved classification performance in previous EEG studies [4]. The S-Transform was used to track alterations in signal magnitude, frequency and phase of selected EEG source as it has been shown to outperform classical techniques based on either frequency or time domain [5]. It is expected that, early $\mathrm{TF}$ detection can help to minimize the affect of TF through visual cues in treatment of PD.

The main contributions of this paper are the novel technique that combined source separator ICA-EBM and EEG feature extraction using S-Transform decomposition. Artificial neural networks classified these extracted features, which were optimized by Bayesian inference techniques. The results of this study suggest that our proposed methodology is a promising non-invasive approach for improving Turning Freeze classification accuracy during ambulation in PD patients.

\section{METHODS}

\section{A. Data Collection and Pre-processing}

EEG data was collected from six PD patients with clinically confirmed freezing of gait in a structured series of Timed Up and Go (TUG) tasks. The patients were recruited from the Parkinson's Disease Research Clinic, Brain and Mind Center, The University of Sydney. This study was approved for by The Human Research and Ethics Committee of the University of Sydney. Assessments were performed in the practically-defined "off" state, with patients having been withdrawn from dopaminergic replacement therapy for more than twelve hours before testing. All subjects demonstrated multiple episodes of freezing during turning in the TUGs 
(66.3\% of turns elicited a TF on average). The EEG was recorded from 15 locations by $\mathrm{Ag} / \mathrm{AgCl}$ scalp electrodes of a Bio-semi Active-Two system. These fifteen locations of interest were chosen based on our previous findings, which were reported as the most affected channels underlying Freezing of Gait in PD patients [7]. These electrodes were positioned over the following key cortical regions: F3, F4, $\mathrm{FC} 1, \mathrm{FC} 2, \mathrm{C} 3, \mathrm{C} 4, \mathrm{CP} 1, \mathrm{CP} 2, \mathrm{CZ}, \mathrm{P} 3, \mathrm{P} 4, \mathrm{PZ}$ and $\mathrm{O} 1, \mathrm{O} 2$, $\mathrm{OZ} \quad(\mathrm{F}=$ frontal, $\mathrm{C}=$ central, $\mathrm{P}=$ parietal, $\mathrm{O}=$ occipital and $\mathrm{Z}=$ midline). The references were taken by averaging the two electrodes A1 and A2, which were placed on the ear lobes. The recording was segmented to 1 -second durations and the sampling rate was $512 \mathrm{~Hz}$.

Two separate conditions were identified for each patient based on video recordings of the TUGs, which is currently used as the golden standard to characterize FOG [3]

i) Normal Turning (NT): identified as a 2 second epoch of time in which a patient successfully made a right or left turn (180 degrees or 540 degrees) inside a taped $1 \mathrm{~m}^{2}$ box on the floor.

ii) Turning Freezing (TF): identified as an epoch of time in which patients suddenly became unable to make a turn inside a taped $1 \mathrm{~m}^{2}$ box on the floor, despite the intention to do so. The duration of TF episodes normally occur from 2 seconds to 5 seconds.

In this study, data from six PD patients resulted in 204 seconds of EEG data samples of TF which were matched to 204 seconds of EEG data samples of NT to be used for further analyses. Data were filtered using band-pass filter $(0.5-40 \mathrm{~Hz})$. Artifacts such as eye activity movement and heart rate signals were removed by Automatic Artifact Removal (AAR) using the EEGLab toolbox. In order to control inherent differences between electrodes and individual subjects, a Z-transformation was applied to normalize EEG data.

\section{B. Feature Extraction using S-Transform}

The S-transform is a time-frequency analysis technique proposed by Stockwell, Manishina and Lowe in 1996 [5]. The S-transform was developed on the basis of short time Fourier Transform (STFT) and continuous wavelet transform (WT) involving direct time-frequency mapping. The advantages of the S-transform are its linearity, lossless reversibility, multi-resolution and good time-frequency resolution. The S-Transform generates a constant relative bandwidth analysis while maintaining a direct link with the Fourier spectrum. In this study, four frequency bands were analyzed, namely: theta $(4-8 \mathrm{~Hz})$, alpha $(8-13 \mathrm{~Hz})$, low beta $(1 \beta, 13-21 \mathrm{~Hz})$ and high beta $(\mathrm{h} \beta, 21-38 \mathrm{~Hz})$.

Given $\mathrm{h}(\mathrm{t})$ is a continuous signal, the S-transform is defined as:

$$
S(\tau, f)=\int_{-\infty}^{\infty} h(t) w(\tau-t, f) \exp (-i 2 \pi f t) d t
$$

where $w(\tau-t, f)$ denotes the specific mother wavelet of the signal

$$
w(\tau-t, f)=\frac{|f|}{\sqrt{2 \pi}} \exp \left(-0.5(\tau-t)^{2} f^{2}\right)
$$

where $f$ denotes the frequency, $t$ denotes the time and $\tau$ denotes the delay with

$$
\int_{-\infty}^{\infty} w(t, f) d t=1
$$

Here, two parameters were extracted: the first is the maximum amplitude for each band at a time $(\mathrm{t}), A^{\max }(t)$. The second parameter was computed as the sum of amplitude of the each band at a time ( $(\mathrm{t}), A^{\text {sum }}(t)$. These main features were used to explore the neural correlates underlying the episodes of turning freezing.

\section{Source separation using ICA-EBM and input for classification}

For improving classification results for TF detection, several pre-processing steps were performed prior to feeding the data to our classifier. ICA-EBM was chosen as a source separator to our EEG data because it has been shown to improve classification result in some previous EEG studies [4]. At first, our EEG data were fed to the source separation ICA-EBM for further analysis. Its aim is to separate the mixed information into independent components. ICA-EBM provides flexible density matching through the use of contrast functions based on the maximum entropy principle [6]. ICAEBM can be applied to separate sources that are both sub- or super-Gaussian distributions using only a small class of nonlinear functions. The algorithm adopted a line search procedure and initially used updates that constrain the ICA de-mixing matrix to be orthogonal for robust performance [6]. The next step included a feature extraction module that transformed the pre-processed EEG signal into S-Transform decomposition based on 4 sub-bands. Only useful features with corrected $p$-values $<0.05$, as computed by the nonparametric Wilcoxon Signed Rank Test, were employed as input for our classifier.

\section{Classification}

For the classification algorithm, Bayesian neural networks (BNN) were implemented. Bayesian regularization framework has been proposed to improve the generalization abilities of neural networks regardless of finite and noisy obtainable data [4]. Applying Bayesian techniques to neural network training and prediction offers principle methods for determining optimal weight decay coefficients and model selection while making efficient use of training data. The use of the hyper parameters in the cost function can prevent the network from being trapped in poor generalization. As a result, a validation data set is not needed for Bayesian training. This is highly suitable for experiments such as ours where a limited set of data is only available for training and testing.

The BNN structure uses a 3-layer (input, hidden and output layers) feed-forward structure as follows:

$$
z(x, w)=f_{1}\left(b_{k}+\sum_{j=1}^{m} \bar{w}_{k j} f_{2}\left(b_{j}+\sum_{i=1}^{n} w_{j i} x_{n}\right)\right)
$$


where $f_{1}, f_{2}$ denotes the activation function, $x$ presents the input vector, $w$ denotes the weight matrix vector; $w_{j i}$ i denotes the weight of the link between the $i$ hidden node and the $j$ input; $\bar{w}_{k j}$ denotes the weight of the link between $i$ hidden node and the output, $b_{\mathrm{k}}$ and $b_{\mathrm{j}}$ denote the biases; $m$ denotes the number of outputs; $n$ denotes the number of inputs.

The BNN training modifies the objective function of the networks such as the sum of squared error (mse)

$$
F=E_{d}=\frac{1}{I} \sqrt{\sum_{i=1}^{n}\left(d_{i}-o_{i}\right)^{2}}
$$

where $E_{d}$ is the error function, $d_{i}$ is the desired output, and $o_{i}$ is the actual output.

The regularization improves the model's generalization by adding the sum of squared weight function $E_{w}$ to the objective function component $(w)$ :

$$
F(w)=\beta E_{d}+\alpha E_{w}
$$

where $\beta$ and $\alpha$ are two hyper-parameters which indicate minimal error, and minimal weights to seek in the learning process.

This addition is proposed to find best generalization by optimizing these parameters in the Bayesian framework [4, 6]. To improve the efficiency of the optimization, Bayesian was added to the Levenberg-Marquardt and to be used for the Gauss-Newton approximation to the Hessian matrix, available in this optimization algorithm for learning.

For Bayesian neural network classification, a validation set is not required in a neural network training procedure. As a result, the current dataset was randomly divided into a training set and a test set with each containing $50 \%$ of the original data from all six patients. The input included the sum amplitude $A^{\text {sum }}(t)$ and the maximum amplitude $A^{\max }(t)$ obtained from the EEG signals based on four sub-bands from two events (NT and TF). In comparison, the features were extracted by S-Transform in both cases, with ICA-EBM and without ICA-EBM were used for classification. The desired output was set at 1 in cases of TF and 0 in cases of NT. This system is expected to test with the new patients in the future.

\section{RESULTS}

\section{A. Feature Extraction Results}

Figure 1 show the time-frequency distributions of Stransform based on the above EEG signals from the frontal F4 electrode of one PD patient. The NT segments in run from 0 to 5 seconds and the TF segments run from 6 to 10 seconds. As clearly demonstrated, there are significant increases during TF across theta, alpha, low beta and specifically high

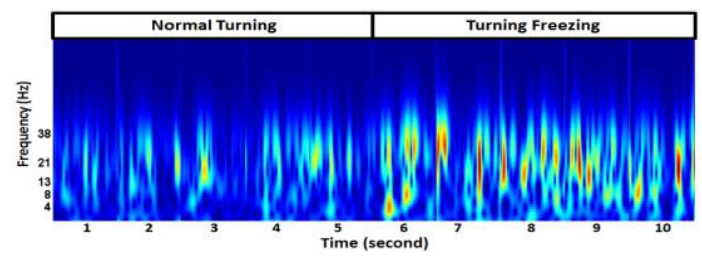

Figure 1. Time-frequency distribution in NT( 1-5s), TF ( 6-10s) in F4.
TABLE I.

\begin{tabular}{|c|c|c|c|c|}
\hline Location & Band & Normal Turning & Turning Freezing & Significant \\
\hline & & $A^{\text {mean }}(t) \pm \mathrm{std}$ & $A^{\text {mean }}(t) \pm$ std & \\
\hline \multirow{2}{*}{ F3 } & $1 \beta$ & $0.26 \pm 0.09$ & $0.29 \pm 0.09$ & $* *$ \\
\hline & $\mathrm{h} \beta$ & $0.29 \pm 0.10$ & $0.34 \pm 0.08$ & $* * *$ \\
\hline \multirow{2}{*}{ F4 } & $1 \beta$ & $0.22 \pm 0.09$ & $0.26 \pm 0.09$ & $* *$ \\
\hline & $h \beta$ & $0.26 \pm 0.10$ & $0.32 \pm 0.09$ & $* * *$ \\
\hline $\mathrm{FC} 1$ & $\mathrm{~h} \beta$ & $0.32 \pm 0.09$ & $0.36 \pm 0.09$ & $* *$ \\
\hline $\mathrm{FC} 2$ & $\mathrm{~h} \beta$ & $0.30 \pm 0.09$ & $0.35 \pm 0.09$ & $* * *$ \\
\hline C3 & $\mathrm{h} \beta$ & $0.33 \pm 0.09$ & $0.37 \pm 0.09$ & $* * *$ \\
\hline \multirow{2}{*}{$\mathrm{C} 4$} & $1 \beta$ & $0.24 \pm 0.10$ & $0.27 \pm 0.10$ & $*$ \\
\hline & $\mathrm{h} \beta$ & $0.27 \pm 0.09$ & $0.31 \pm 0.09$ & $* *$ \\
\hline CP1 & $\mathrm{h} \beta$ & $0.32 \pm 0.09$ & $0.37 \pm 0.09$ & $* *$ \\
\hline $\mathrm{CP} 2$ & $\mathrm{~h} \beta$ & $0.32 \pm 0.09$ & $0.36 \pm 0.08$ & $* *$ \\
\hline \multirow{2}{*}{$\mathrm{CZ}$} & $1 \beta$ & $0.29 \pm 0.09$ & $0.33 \pm 0.10$ & $* *$ \\
\hline & $\mathrm{h} \beta$ & $0.32 \pm 0.09$ & $0.37 \pm 0.10$ & $* * *$ \\
\hline P3 & $\mathrm{h} \beta$ & $0.36 \pm 0.11$ & $0.41 \pm 0.10$ & $* *$ \\
\hline P4 & $\mathrm{h} \beta$ & $0.33 \pm 0.10$ & $0.37 \pm 0.09$ & $* *$ \\
\hline $\mathrm{Pz}$ & $\mathrm{h} \beta$ & $0.35 \pm 0.10$ & $0.40 \pm 0.09$ & $* * *$ \\
\hline \multirow{4}{*}{ O1 } & $\theta$ & $0.30 \pm 0.12$ & $0.33 \pm 0.08$ & $*$ \\
\hline & $\alpha$ & $0.58 \pm 0.22$ & $0.66 \pm 0.18$ & $* *$ \\
\hline & $1 \beta$ & $0.51 \pm 0.21$ & $0.59 \pm 0.16$ & $* * *$ \\
\hline & $\mathrm{h} \beta$ & $0.44 \pm 0.15$ & $0.51 \pm 0.12$ & $* * *$ \\
\hline \multirow{3}{*}{$\mathrm{OZ}$} & $\alpha$ & $0.59 \pm 0.23$ & $0.67 \pm 0.18$ & $* *$ \\
\hline & $1 \beta$ & $0.51 \pm 0.21$ & $0.60 \pm 0.15$ & $* * *$ \\
\hline & $\mathrm{h} \beta$ & $0.43 \pm 0.14$ & $0.50 \pm 0.11$ & $* * *$ \\
\hline \multirow{4}{*}{$\mathrm{O} 2$} & $\theta$ & $0.30 \pm 0.12$ & $0.32 \pm 0.10$ & * \\
\hline & $\alpha$ & $0.58 \pm 0.25$ & $0.65 \pm 0.20$ & * \\
\hline & $1 \beta$ & $0.48 \pm 0.22$ & $0.58 \pm 0.17$ & $* * *$ \\
\hline & $h \beta$ & $0.40 \pm 0.15$ & $0.48 \pm 0.11$ & $* * *$ \\
\hline
\end{tabular}

FEATURE EXTRACTION

*: Significant at $0.001<p \leq 0.05$ and Cohen's $d<0.3$

**: Significant at $0.0001<p \leq 0.001$ and Cohen's $0.3 \leq d<0.4$

***: Significant at $p \leq 0.0001$ and Cohen's $d \geq 0.4$

beta frequency bands.

To investigate significant differences between periods of NT and periods of TF, a Wilcoxon Signed-Rank Test was conducted on the sample of 6 patients. Table 1 shows the significant maximum amplitude $\left(A^{\max }(t)\right)$ in the four subbands between periods of NT and TF. Smaller p-values ( $p$ values $\leq 0.0001)$ and larger Cohen's $d(d \geq 0.4)$ indicated the biggest differences in features between the two conditions.

The largest significant increase in activity was found in low beta and high beta across the brain regions during TF. The strongest maximum differences were found in high beta activity from frontal $\mathrm{F} 4 \quad(\mathrm{~d}=0.6143)$ and occipital $\mathrm{O} 2$ $(\mathrm{d}=0.5919)$ locations. Moreover, maximum alpha band activity increased significantly only in occipital locations $\mathrm{O}$, $\mathrm{O} 2$ and $\mathrm{Oz}$ during $\mathrm{TF}$ stages. Theta band increased remarkably only in occipital locations $\mathrm{O} 1, \mathrm{O} 2$ during TF.

\section{B. Source Separator ICA-EBM and Classification results}

In this stage, EEG data was fed into the ICA-EBM, which resulted in ICA separated sources of EEG data. Based on our previous finding, the additional optimal systems comprised four locations F3, C4, P4 and O2, which were selected for TF detection [7]. These optimized ICA data sources were further segmented for feature extraction using the S-Transform. The mean and maximum amplitude from four EEG frequency bands of each location were used to evaluate their ability in detecting TF. These significant features with $p$-values $\leq 0.05$ were used as the main parameters for detecting TF events in PD patients.

Classification results for $\mathrm{TF}$ events detection using 
TABLE II. CLASSIFICATION RESULTS

\begin{tabular}{ccccccccc}
\hline Cha & ICA & H & \multicolumn{3}{c}{ Training } & \multicolumn{3}{c}{ Testing } \\
\hline & & & Sen & Spe & Acc & Sen & Spec & Acc \\
\hline 15 & No & 4 & $79.8 \%$ & $78.7 \%$ & $79.2 \%$ & $79.1 \%$ & $77.1 \%$ & $78.2 \%$ \\
15 & Yes & 4 & $\mathbf{8 4 . 6 \%}$ & $\mathbf{8 8 . 0 \%}$ & $\mathbf{8 6 . 3 \%}$ & $\mathbf{8 3 . 0} \%$ & $\mathbf{8 7 . 6 \%}$ & $\mathbf{8 5 . 4 \%}$ \\
\hline 4 & No & 7 & $76.3 \%$ & $72.3 \%$ & $74.2 \%$ & $75.7 \%$ & $70.3 \%$ & $73.3 \%$ \\
4 & Yes & 7 & $\mathbf{8 5 . 8 \%}$ & $\mathbf{8 8 . 0 \%}$ & $\mathbf{8 6 . 8 \%}$ & $\mathbf{8 4 . 2 \%}$ & $\mathbf{8 8 . 0 \%}$ & $\mathbf{8 6 . 2 \%}$ \\
\hline
\end{tabular}

Cha: Number of Channels; ICA: ICA-EBM; H: Hidden Node; Sen:

Sensitivity; Spe: Specificity; Acc: Accuracy

S-Transform decomposition as the feature methods and Bayesian neural networks as the classifier are shown in Table II. The outcome indicated that the S-Transform based feature extraction provided respectable results for TF detection, with $79.1 \%$ of sensitivity, $77.1 \%$ of specificity and $78.2 \%$ of accuracy for the test set. Interestingly, further improvements were obtained when using the combination of ICA-EBM as the source separator before extracting feature, which improved outcomes to a sensitivity of $83.0 \%$, a specificity of $87.6 \%$ and an accuracy of $85.4 \%$ in detecting TF. Moreover, as we expected, the best performance for TF detection was achieved when using our predefined regions of interest (4 locations) plus ICA-EBM for pre-processing. For this combination the results were a sensitivity of $84.2 \%$, a specificity of $88.0 \%$ and an accuracy of $86.2 \%$.

Figure 2 shows the results in the receiver operating characteristic (ROC) curve and analyses. The bold red colored line represents the ROC curve for the method using the source separation of ICA-EBM. The star blue colored line represents the method without source separation. The ROC curve is a plot of the true positive rate versus the false positive rate utilizing different threshold ratios as a sweeping variable. The areas under the curve (AUC) of ROC were also calculated to quantitatively evaluate classification performance [4]. It can be seen that the method using the inclusion of the source separation of ICA-EBM had a higher AUC of 0.9296 compared to the method without inclusion of the source separation, which had an AUC of 0.8127.

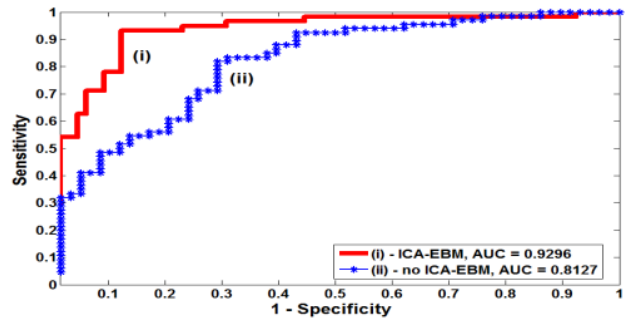

Figure 2. AUC plot

\section{DISCUSSION}

In this paper, $\mathrm{TF}$ was found to be associated with a remarkable increase in high beta activity across the cortex. The most prominent results were found for high beta in the frontal F4 and occipital O2 locations. These findings are in accordance with our previous work using FFT and functional magnetic resonance imaging for analyzing turning behavior in PD patients [2, 3]. Based on these results, we hypothesize that the subthalamic nucleus (STN) of PD patients with freezing, which has been shown to shut down motor activity using high beta frequencies, likely underlies the trigger of TF [2]. Indeed, deep brain stimulation of the STN, specifically when using lower $60 \mathrm{~Hz}$ frequencies can often alleviate FOG in PD [2]. The significant increase in high beta of frontal regions could further implicate the recruitment of a putative stopping network that worsens freezing behavior.

In terms of classification, the application of source separation using ICA-EBM improved the classification performance as compared to the case without source separation. Altogether, the result shows that the combination of the ICA-EBM (source separator) and the S-Transform (feature extraction) significantly improved the sensitivity and specificity of TF detection.

\section{CONCLUSION}

During turning freezing, PD patients showed an increase in beta activities across regions that implicate involvement of the STN and a potential recruitment of a hyper-direct stopping network, which may be manifested pathologically as a freeze as sensorimotor processing becomes more complex. The current study provided additional support toward detecting freezing during turning by using the combination of PCA, ICA-EBM, S-Transform analysis and BNN. This method improved classification results in detecting TF in PD. This novel methodology will help the development of a realtime detection device for different sub-types of FOG in PD. Also, the understanding of underlying neurobiology will ultimately promote the development of novel therapies and technologies to assist with the management of FOG in PD.

\section{ACKNOWLEDGMENT}

The authors would like to dedicate this work to the memory of Dr A.M. Ardi Handojoseno who contributed significantly to our research in Parkinson's disease. His intellect, kindness and compassion will always remain deeply in our hearts.

\section{REFERENCES}

[1] J. D. Schaafsma, Y. Balash, T. Gurevich, A. L. Bartels, J. M Hausdorff, and N. Giladi, "Characterization of freezing of gait subtypes and the response of each to levodopa in Parkinson's disease," European Journal of Neurology, vol. 10, pp. 391-398, 2003.

[2] M. Gilat, J. M. Shine, C. C. Walton, C. O’Callaghan, J. M. Hall, and S. J. G. Lewis, "Brain activation underlying turning in Parkinson's disease patients with and without freezing of gait: a virtual reality fMRI study," Npj Parkinson's Disease, vol. 1, p. 15020, 2015

[3] A. M. A. Handojoseno, M. Gilat, Q. T. Ly, H. Chamtie, J. M. Shine, T. N. Nguyen, et al., "An EEG study of turning freeze in Parkinson's disease patients: The alteration of brain dynamic on the motor and visual cortex," in 2015 37th Annual International Conference of the IEEE Engineering in Medicine and Biology Society (EMBC), 2015 , pp. 6618-6621

[4] R. Chai, G. Naik, T. N. Nguyen, S. Ling, Y. Tran, A. Craig, et al., "Driver Fatigue Classification with Independent Component by Entropy Rate Bound Minimization Analysis in an EEG-based System," IEEE Journal of Biomedical and Health Informatics, vol. PP, pp. 1-1, 2016

[5] R. G. Stockwell, L. Mansinha, and R. P. Lowe, "Localization of the complex spectrum: the S transform," IEEE Transactions on Signal Processing, vol. 44, pp. 998-1001, 1996.

[6] X. L. Li and T. Adali, "Independent Component Analysis by Entropy Bound Minimization," IEEE Transactions on Signal Processing, vol. 58, pp. 5151-5164, 2010

[7] Q. T. Ly, A. M. A. Handojoseno, M. Gilat, N. Nguyen, R. Chai, Y. Tran, H. T. Nguyen et al., "Identifying montages that best detect the electroencephalogram power spectrum alteration during freezing of gait in Parkinson's disease patients," in 2016 38th Annual International Conference of the IEEE Engineering in Medicine and Biology Society (EMBC), 2016, pp. 6094-6097 